\title{
Analysis on Increasing Customer Sales by the Use of Restaurant Recommender System
}

\author{
M. Ashrafa \\ M.O.P Vaishnav \\ College \\ For Women \\ Chennai,India
}

\author{
M. Lalitha \\ M.O.P Vaishnav \\ College \\ For Women \\ Chennai,India
}

\author{
D. Radha \\ M.O.P Vaishnav \\ College \\ For Women \\ Chennai,India
}

\author{
R. Jayaparvathy \\ S.S.N College of \\ Engineering \\ Chennai, India
}

\begin{abstract}
In a restaurant recommender system the main focus is on the user rating or perhaps the customer satisfaction that is significant of all. The contextual features are closely studied which aids as the precipitate for the study. In order to identify relevant contextual information for the study, only those cardinal features that are significant for the decision criteria are considered. Subsequently these features which are identified are used for the analysis. However, insignificant features may not scale up well for the study. This reduces dimensionality issues. Previously this kind of an approach using contextual feature has been utilized for various studies in restaurant recommender system. This is of prime importance in order to build the relevant contextual user profile. The customer rating or the level of satisfaction stated by the customer is used to study the perception of the restaurant by the customer. Also to extract those customers who visit a particular restaurant moderately than in comparison with those who visits very often or visits less than moderate. These customers are extracted to be focused upon for increase of sale by sending them text messages or emails regarding the offers and other discounts which is more economical rather than sending those offers to every customer visiting the restaurant.
\end{abstract}

\section{General Terms}

Restaurant recommender system, customer behavior, clustering, data mining.

\section{Keywords}

Contextual information, feature selection.

\section{INTRODUCTION}

It's common to ask friends about new places to eat or visit the nearby restaurant. Touristic guides are other conventions used to find good restaurants in the nearby vicinity. However, customers having divergent likes and dislikes would not want to have an unpleasant experience after dining in a restaurant so they would expect a recommender system that gives features about a restaurant in a specific locality. Consequently, the suggestions provided by the recommender system would be more precise than those provided by other customers who had previously visited that restaurant. Actually, recommender systems are common online services that help users to cope with plethora of information by reclaiming useful items according to their preferences. A content-based recommendation system builds a model only from the user's favorite items. This is both useful to attract intending customers as well as give the customers a better experience after visiting the restaurant. As this mines the data regarding the particular customer likes and dislikes or rather preferences, the restaurant can increase their sale focusing on specific groups of customers as intended by the restaurant, either moderate, less than moderate or increased frequency of visits by a customer. Sending customized text messages to customers with moderate frequency, increase of sale is possible. These messages can also be sent to every customer registered with the particular restaurant but it does not hold good in terms of economy as sending offers to customers with high frequency of visits is not needful or economically efficient. On the contrary customers with very low frequency of visits need not be focused for increased sale because there are customers who fall in between the very high and very low frequency groups who can potentially be the focus of the restaurant to possibly convert them into the high frequency group in future. Consequently, this aids in increasing sale by using feature selection technique.

\section{LITERATURE REVIEW}

This review throws light upon two main topics i.e. relevant contextual feature information and user profile built upon the significant contextual features. Context-aware recommender systems are on the rise among the potential customers. Although the effect of contextual information has not been thoroughly examined, related research discloses that contextual information is significant for the study to provide increased focus on specific group of customers. The effect of context variables in a restaurant recommender system was analyzed and several clusters were built upon according to the statistic interdependence between matching set of context variables. The anticipative accuracy is enhanced by the context variables. It shows the benefits of contextual information in precision. Another approach that was used in the study consisted of splitting of user profile which results in several sub-profiles, in order to focus more on divergent behaviors. It was showed that accuracy could increase by making recommendations using sub-profiles in place of a single user profile. The integration of contextual information necessitates dimensionality reduction for a clear result. These problems are dealt with data mining techniques. However, when the system returns plethora of results, an algorithm is used to minimize the result list eliminating the insignificant data. These results show that anticipative performance can be imp by a closed selection of contextual features and their corresponding item ratings. In contrast to the reviewed works, in this study, the primary goal is to identify relevant attributes and then assess their effects in the system's anticipative performance. This serves as a semantic restaurant recommender system that blends social and contextual aspects that was used as the test base. 


\section{SYSTEM MODEL}

\subsection{Algorithm Restaurant Recommender System \\ START}

Set Customer_preferences;

Set Nearest_Restaurant_Availabilities;

Display Customer_preferences that match the Nearest_Restaurant-Availabilities;

Set Customer_Frequency as Very_Low $=0$, Moderate $=1$, High=2;

Display Customers with Customer_Frequency $=1$;

Send Customized_Text_Messages to Customers with END

Customer_Frequency $=1$;

The algorithm extracts the customers whose preferences meet the restaurant features and also specially mines those customers whose frequency of visiting the restaurant is moderate. In order to increase the sale these customers are considered to be the target group by sending customized text messages regarding the offers and other discounts.

\section{PERFORMANCE AND ANALYSIS}

From figure (1) we can analyze the customer behavior by inputting customer data holding the customer ratings for the restaurants into WEKA tool. The result shows the clusters of varied customer preferences. By analyzing the data using the restaurant recommender system we can extract those customers who have given moderate ratings and study their preferences and implement innovations in the restaurant as preferred by those customers in order to increase the sale.

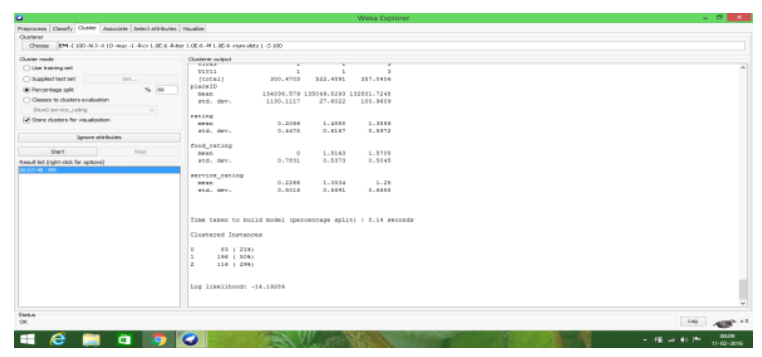

Figure (1) showing input of customer data using WEKA tool

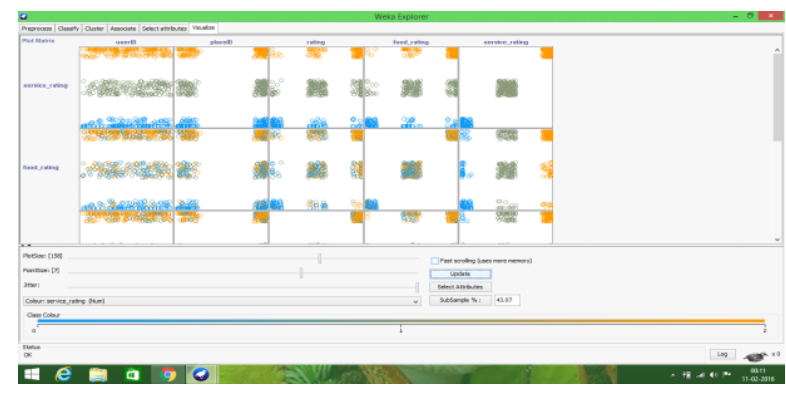

Figure (2) showing the results of Cluster Analysis using WEKA tool

\section{CONCLUSION AND FUTURE ENHANCEMENT}

This study focuses on increasing the sale by achieving both customer satisfaction and increased attention for specific group of customers who are potential customers, this is done by sending text messages to that specific group so that there is a possibility of converting their visits from moderate to high. Further enhancements can be made to study more about the customer behavior of a specific group to further increase sales by creation of innovative ideas to also include those features that are trending in particular vicinity.

\section{REFERENCES}

[1] Adomavicius, R. Sankaranarayanan, S. Sen, and A. Tuzhilin. Incorporating contextual information in recommender systems using a multidimensional approach. ACM Transactions on Information Systems, 23:103-145, January 2005.

[2] L. Baltrunas and X. Amatriain. Towards time-dependant recommendation based on implicit feedback. In RecSys'09: Workshop on context-aware systems (CARS-2009), 2009.

[3] L. Baltrunas and F. Ricci. Locally adaptive neighborhood selection for collaborative filtering recommendations. In Proceedings of the 5th international conference on Adaptive Hypermedia and Adaptive Web-Based Systems, pages 22-31, Berlin, Heidelberg, 2008. Springer-Verlag

[4] R. Ponce-Medell' in, J. G. Gonz'alez-Serna, and L. Ruiz. Technology integration around the geographic information: A state of the art. International Journal of Computer Science Issues, 5:17-26, 2009.

[5] Fan Yang and Zhi-Mei Wang. A Mobile Location-based Information Recommendation System Based on GPS and WEB2.0 Services. In WSEAS Transactions on Computers, 2009, Pages 725-734.

[6] Chumki Basu, Haym Hirsh and William Cohen. Recommendation as Classification: Using Social and Content-Based Information in Recommendation. In AAAI-98 Proceedings, 1998.

[7] Kalseth, Fredrik: "Developing a Restaurant Recommender System”, UC Santa Cruz, May 2005.

[8] Burke, Robin: "Knowledge-based Recommender System”, UC Irvine, 2000.

[9] R. Burke. Integrating knowledge-based and collaborative-filtering recommender systems. Papers from The AAAI Workshop on Artificial Intelligence for Electronic Commerce WS-99-01, AAAI Press, Menlo Park, California, 1999. 